\title{
Hyperfine splitting of P-states in light muonic ions
}

\author{
A.E. Dorokhov ${ }^{1,2, *}$, A.P. Martynenko ${ }^{2, * *}$, F.A. Martynenko ${ }^{2, * * *}$, and O.S. Sukhorukova ${ }^{2, * * * *}$ \\ ${ }^{1}$ Joint Institute of Nuclear Research, BLTP, 141980, Moscow region, Dubna, Russia \\ ${ }^{2}$ Samara National Research University, Moskovskoye Shosse 34, 443086, Samara, Russia
}

\begin{abstract}
We calculate hyperfine structure intervals for P-states in muonic ions of lithium, beryllium and boron. To construct the particle interaction operator in momentum space we use the tensor method of projection operators on states with definite quantum numbers of total atomic momentum $F$ and total muon momentum $j$. We take into account vacuum polarization, relativistic, quadruple and structure corrections of orders $\alpha^{4}, \alpha^{5}$ and $\alpha^{6}$. The obtained numerical values of hyperfine splittings can be used for a comparison with future experimental data.
\end{abstract}

\section{Introduction}

One of the future scientific directions of CREMA (Charge Radius Experiments with Muonic Atoms) collaboration is related with light muonic atoms of lithium, beryllium and boron $[1,2]$. Such experiments on measurement of fine and hyperfine structure could obtain new values of charge radii of $\mathrm{Li}, \mathrm{Be}$ and $\mathrm{B}$. The program of experiments with light muonic atoms was discussed many years ago in $[3,4]$, where the estimation of different energy intervals in the leading order was performed. In our recent papers $[5,6]$ we calculated some corrections to the Lamb shift (2P-2S) and hyperfine splitting of S-states in muonic lithium, beryllium and boron and obtain more precise values of these energy intervals. This work continues our investigation in [5-7] to the case of P-wave part of the spectrum. The account of hyperfine structure of P-levels is also necessary because experimental transition frequencies are measured between different components of $2 \mathrm{P}$ and $2 \mathrm{~S}$ states. Despite the fact that general theoretical methods for the calculation of hyperfine structure of P-states are well developed, specific numerical calculations of different hyperfine structure intervals in the case of P-states in muonic lithium, beryllium and boron were not considered in detail. So, the work [3] contains only general formula of hyperfine structure in leading order. Therefore, the aim of this work is to calculate hyperfine splitting intervals for P-states in muonic $\mathrm{Li}, \mathrm{Be}, \mathrm{B}$ with the account of corrections to vacuum polarization and nuclear structure.

\section{General formalism}

Among nuclei of $\mathrm{Li}, \mathrm{Be}, \mathrm{B}$ there are nuclei of different spins. For the nuclei with $\operatorname{spin} I=s_{2}=$ 1 our approach to the calculation of hyperfine structure was developed in [8] by the example

\footnotetext{
*e-mail: dorokhov@theor.jinr.ru

**e-mail: a.p.martynenko@samsu.ru

***e-mail: f.a.martynenko@gmail.com

****e-mail: o.skhrkv@gmail.com
} 
of muonic deuterium. All nuclei of lithium, beryllium and boron have isotopes with spin $s_{2}=3 / 2$ so we describe further hyperfine structure of such muonic ions which consists of six states: $2^{3} P_{1 / 2}, 2^{5} P_{1 / 2}, 2^{1} P_{3 / 2}, 2^{3} P_{3 / 2}, 2^{5} P_{3 / 2}, 2^{7} P_{3 / 2}$, where the lower index corresponds to muon total momentum $\mathbf{j}=\mathbf{s}_{1}+\mathbf{L}$ and upper index is the factor $(2 F+1)\left(\mathbf{F}=\mathbf{j}+\mathbf{s}_{2}\right)$. The contribution of the leading order $\alpha^{4}$ to HFS of P-states is determined by the amplitude of one-photon interaction which is denoted $T_{1 \gamma}$. In momentum representation the wave function of $2 \mathrm{P}$-state can be written in the tensor form:

$$
\psi_{2 P}(\mathbf{p})=\left(\varepsilon \cdot n_{p}\right) R_{21}(p)
$$

where $\varepsilon_{\delta}$ is the polarization vector of orbital motion, $n_{p}=(0, \mathbf{p} / p), R_{21}(p)$ is the radial wave function in momentum space. Then the contribution to the energy spectrum is determined by the integral:

$$
\Delta E^{h f s}=\int\left(\varepsilon^{*} \cdot n_{q}\right) R_{21}(q) \frac{d \mathbf{q}}{(2 \pi)^{3 / 2}} \int\left(\varepsilon \cdot n_{p}\right) R_{21}(p) \frac{d \mathbf{p}}{(2 \pi)^{3 / 2}} \Delta V^{h f s}(\mathbf{p}, \mathbf{q}) .
$$

The hyperfine potential $\Delta V^{h f s}$ can be constructed by means of one-photon interaction amplitude $T_{1 \gamma}$ using the method of projection operators on states with definite quantum numbers [8-12]. These projection operators can be written in terms of particle wave functions at the rest frame in covariant form. They allow us to avoid direct cumbersome multiplication of different factors in the amplitudes and use the computer methods for calculating amplitudes and the energy levels [13]. For their construction we use two different schemes of momentum adding: 1. $\mathbf{J}=\mathbf{s}_{1}+\mathbf{L}, \mathbf{F}=\mathbf{J}+\mathbf{s}_{2}, 2 . \mathbf{S}=\mathbf{s}_{1}+\mathbf{s}_{2}, \mathbf{F}=\mathbf{S}+\mathbf{L}$. Taking into account that the nuclei with spin $3 / 2$ are described in the Rarita-Schwinger formalism by the spin-vector $v_{\alpha}(p)$ we can write the one-photon interaction amplitude in the form:

$$
\begin{aligned}
T_{1 \gamma}(\mathbf{p}, \mathbf{q})=4 \pi Z \alpha\left(\varepsilon^{*} \cdot n_{q}\right)\left[\bar{u}\left(q_{1}\right)\left(\frac{\left(p_{1}+q_{1}\right)_{\mu}}{2 m_{1}}+\left(1+a_{\mu}\right) \sigma_{\mu \epsilon} \frac{k_{\epsilon}}{2 m_{1}}\right) u\left(p_{1}\right)\right]\left(\varepsilon \cdot n_{p}\right) D_{\mu \nu}(k) \times \\
\bar{v}_{\alpha}\left(p_{2}\right)\left\{g_{\alpha \beta} \frac{\left(p_{2}+q_{2}\right)_{v}}{2 m_{2}} F_{1}\left(k^{2}\right)+g_{\alpha \beta} \sigma_{v \lambda} \frac{k^{\lambda}}{2 m_{2}} F_{2}\left(k^{2}\right)+\right. \\
\left.\frac{k_{\alpha} k_{\beta}}{4 m_{2}^{2}} \frac{\left(p_{2}+q_{2}\right)_{v}}{2 m_{2}} F_{3}\left(k^{2}\right)+\frac{k_{\alpha} k_{\beta}}{4 m_{2}^{2}} \sigma_{v \lambda} \frac{k^{\lambda}}{2 m_{2}} F_{4}\left(k^{2}\right)\right\} v_{\beta}\left(q_{2}\right),
\end{aligned}
$$

where $p_{1,2}=\frac{m_{1,2}}{\left(m_{1}+m_{2}\right)} P \pm p$ are four-momenta of initial muon and nuclear, $q_{1,2}=\frac{m_{1,2}}{\left(m_{1}+m_{2}\right)} Q \pm q$ are four-momenta of final muon and nuclear, $a_{\mu}$ is the muon anomalous magnetic moment. They are expressed in terms of total two-particle momenta $P, Q$ and relative momenta $p, q$. $D_{\mu \nu}(k)$ is the photon propagator which is taken to be in the Coulomb gauge. Four form factors which parametrise the nucleus electromagnetic current can be expressed through multipole form factors measured in experiments: charge $G_{E 0}$, electroquadrupole $G_{E 2}$, magnetic-dipole $G_{M 1}$ and magnetic-octupole $G_{M 3}$ form factors [14-16]. The nucleus spin-vector wave function is described by

$$
\psi_{\mu, \alpha}(\mathbf{p}, \sigma)=\sum_{\lambda, \omega}<\frac{1}{2} \omega ; 1 \lambda \mid \frac{3}{2} \sigma>\varepsilon_{\mu}(\mathbf{p}, \lambda) u_{\alpha}(\mathbf{p}, \omega)
$$

where $<\frac{1}{2} \omega ; 1 \lambda \mid \frac{3}{2} \sigma>$ are the Clebsch-Gordan coefficients. In order to obtain the contribution to HFS of order $\alpha^{4}$ including recoil effects we should know also the transformation law of spin-vector to the rest frame. 
To describe the hyperfine structure of state $2 P_{1 / 2}$ we introduce in (3) on the first stage of the transformation the projection operator on the muon state with $j=1 / 2$ :

$$
\hat{\Pi}_{j=1 / 2}=\left[u(0) \varepsilon_{\omega}(0)\right]_{j=1 / 2}=\frac{1}{\sqrt{3}} \gamma_{5}\left(\gamma_{\omega}-v_{\omega}\right) \psi(0),
$$

where $\psi(0)$ is the Dirac spinor describing the muon state with $\mathrm{j}=1 / 2, v=(1,0,0,0)=P /\left(m_{1}+\right.$ $\left.m_{2}\right)$ is the auxiliary four vector. On the second stage we should project muon-nucleus pair on state with total momentum $F=2$ or $F=1$. In the case of state with $F=2$ the projection operator has the form

$$
\hat{\Pi}_{j=1 / 2}(F=2)=\left[\psi(0) \bar{v}_{\alpha}(0)\right]_{F=2}=\frac{1+\hat{v}}{2 \sqrt{2}} \gamma_{\tau} \varepsilon_{\alpha \tau},
$$

where the tensor $\varepsilon_{\alpha \tau}$ describes the state $F=2$. Then the averaged over the projections $M_{F}$ amplitude takes the form:

$$
\begin{gathered}
\left.\overline{T_{1 \gamma}(\mathbf{p}, \mathbf{q})}\right)_{F=2}^{j=1 / 2}=\frac{Z \alpha}{5} n_{q}^{\delta} n_{p}^{\omega} \operatorname{Tr}\left\{\gamma_{\sigma} \frac{1+\hat{v}}{2 \sqrt{2}}\left(\gamma_{\delta}-v_{\delta}\right) \gamma_{5} \frac{\left(\hat{q}_{1}+m_{1}\right)}{2 m_{1}} \Gamma_{\mu} \frac{\left(\hat{p}_{1}+m_{1}\right)}{2 m_{1}} \gamma_{5}\left(\gamma_{\omega}-v_{\omega}\right) \times\right. \\
\left.\frac{1+\hat{v}}{2 \sqrt{2}} \gamma_{\rho} \frac{\left(\hat{p}_{2}-m_{2}\right)}{2 m_{2}} \Gamma_{\alpha \beta}^{v} \frac{\left(\hat{q}_{2}-m_{2}\right)}{2 m_{2}}\right\} D_{\mu \nu}(k) \hat{\Pi}_{\beta_{1} \sigma, \alpha_{1} \rho} L_{\alpha \alpha_{1}} L_{\beta \beta_{1}}, \sum_{M_{F}=-2}^{2} \varepsilon_{\beta \lambda}^{*} \varepsilon_{\alpha \rho}=\hat{\Pi}_{\beta \lambda, \alpha \rho},
\end{gathered}
$$

where we introduce for the convenience short designations of nucleus vertex function

$\Gamma_{\alpha \beta}^{v}=\left[g_{\alpha \beta} \frac{\left(p_{2}+q_{2}\right)_{v}}{2 m_{2}} F_{1}\left(k^{2}\right)+g_{\alpha \beta} \sigma_{v \lambda} \frac{k^{\lambda}}{2 m_{2}} F_{2}\left(k^{2}\right)+\frac{k_{\alpha} k_{\beta}}{4 m_{2}^{2}} \frac{\left(p_{2}+q_{2}\right)_{v}}{2 m_{2}} F_{3}\left(k^{2}\right)+\frac{k_{\alpha} k_{\beta}}{4 m_{2}^{2}} \sigma_{v \lambda} \frac{k^{\lambda}}{2 m_{2}} F_{4}\left(k^{2}\right)\right]$,

$L_{\alpha \alpha_{1}}$ are the Lorentz factors of vector fields, the lepton vertex function

$$
\Gamma_{\mu}=\frac{p_{1, \mu}+q_{1, \mu}}{2 m_{1}}+\left(1+a_{\mu}\right) \sigma_{\mu \epsilon} \frac{k_{\epsilon}}{2 m_{1}} .
$$

We introduce in (7) the factor $3 / 4 \pi$ connected with the normalization condition of polarization vector in (1). The remaining cumbersome part of calculating the trace and numerous convolutions by the Lorentz indices can be performed by means of the package Form [13]. As a result we obtain the muon-nucleus interaction operator for the state $2^{5} P_{1 / 2}$ in the form:

$$
\begin{gathered}
V_{1 \gamma}(\mathbf{p}, \mathbf{q})_{F=2}^{j=1 / 2}=\frac{2 \alpha \mu_{N}}{27 m_{1} m_{p}(\mathbf{p}-\mathbf{q})^{2}}\left\{\frac{9}{2} p q+\frac{9 m_{1}}{4 m_{2}} p q-\frac{9}{4}(\mathbf{p q})\left(\frac{p}{q}+\frac{q}{p}\right)+\frac{27 m_{1}}{8 m_{2}}(\mathbf{p q})\left(\frac{p}{q}+\frac{q}{p}\right)(10)\right. \\
-\frac{9 m_{1}}{m_{2}} \frac{(\mathbf{p q})^{2}}{p q}+a_{\mu}\left[\frac{9}{4} p q-\frac{9}{4}(\mathbf{p q})\left(\frac{p}{q}+\frac{q}{p}\right)+\frac{9}{4} \frac{(\mathbf{p q})^{2}}{p q}\right]+\frac{a_{\mu}}{F_{2}(0)}\left[-\frac{27}{4} p q\left(1+\frac{m_{2}}{m_{1}}\right)+\frac{27}{4} \frac{(\mathbf{p q})^{2}}{p q}+\right. \\
\left.\frac{27 m_{2}}{8 m_{1}}(\mathbf{p q})\left(\frac{p}{q}+\frac{q}{p}\right)\right]+\frac{27(\mathbf{p q})\left(\mathbf{p}^{2}-\mathbf{q}^{2}\right)^{2}}{8(\mathbf{p}-\mathbf{q})^{2} F_{2}(0) p q}-\frac{27}{8 F_{2}(0)}\left[p q\left(2+\frac{m_{1}}{m_{2}}+\frac{m_{2}}{m_{1}}\right)-\frac{m_{2}}{m_{1}}(\mathbf{p q})\left(\frac{p}{q}+\frac{q}{p}\right)\right. \\
\left.\left.+(\mathbf{p q})\left(\frac{p}{q}+\frac{q}{p}\right)+4 m_{1} m_{2} \frac{(\mathbf{p q})}{p q}-\frac{2 m_{1}}{m_{2}} \frac{(\mathbf{p q})^{2}}{p q}\right]\right\},
\end{gathered}
$$

where we set $F_{1}(0)=1$. This expression clearly shows the general structure of potentials for various states, which we obtain at the exit from Form. It is important to note that when constructing potentials in this way, we obtain not only the hyperfine part of the potentials, but also the Coulomb contributions and contributions to the fine structure, which are further 
reduced when considering hyperfine splitting. Let us consider also the construction of the potential in the case of $2^{3} P_{1 / 2}$ state. To introduce projection operators for the state $F=1$, $j=1 / 2$, it is necessary to add the spin of the nucleus $s_{2}=3 / 2$ and the total moment of the muon $j=1 / 2$. For this we use a basis transformation of the following form:

$$
\Psi_{S_{2}=3 / 2, F=1, M_{F}}=\sqrt{\frac{2}{3}} \Psi_{\tilde{S}=0, F=1, M_{F}}+\sqrt{\frac{1}{3}} \Psi_{\tilde{S}=1, F=1, M_{F}},
$$

where the state with $s_{2}=3 / 2$ is represented as the sum of two moments $\tilde{s}_{2}=1 / 2$ and $l_{2}=1$, $\tilde{S}=s_{1}+\tilde{s}_{2}$. Further, when working with $\Psi_{\tilde{S}=0, F=1, M_{F}}$ and $\Psi_{\tilde{S}=1, F=1, M_{F}}$ we introduce projection operators on these states, the form of which is well known:

$$
\hat{\Pi}_{\alpha}(\tilde{S}=0, F=1)=\frac{1+\hat{v}}{2 \sqrt{2}} \gamma_{5} \varepsilon_{\alpha}, \hat{\Pi}_{\alpha}(\tilde{S}=1, F=1)=\frac{1+\hat{v}}{4} \gamma_{\sigma} \varepsilon_{\alpha \sigma \rho \omega} \nu^{\rho} \varepsilon^{\omega},
$$

where the polarization vector $\varepsilon^{\omega}$ in right part of (12) describes the state with $F=1$. When using expansion (11), several contributions to the interaction potential of particles in the state $F=1$ arise, which are determined by two expressions for $\tilde{S}=0$ and $\tilde{S}=1$ with weight factors $2 / 3$ and $1 / 3$ respectively:

$$
\begin{aligned}
& {\overline{T_{1 \gamma}(\mathbf{p}, \mathbf{q})}}_{F=1}^{j=1 / 2}(\tilde{S}=0)=\frac{Z \alpha}{3} n_{q}^{\delta} n_{p}^{\omega} \operatorname{Tr}\left\{\gamma_{5} \frac{1+\hat{v}}{2 \sqrt{2}}\left(\gamma_{\delta}-v_{\delta}\right) \gamma_{5} \frac{\left(\hat{q}_{1}+m_{1}\right)}{2 m_{1}} \Gamma_{\mu} \frac{\left(\hat{p}_{1}+m_{1}\right)}{2 m_{1}} \gamma_{5}\left(\gamma_{\omega}-v_{\omega}\right) \times\right. \\
& \frac{1+\hat{v}}{2 \sqrt{2}} \gamma_{5} \frac{\left(\hat{p}_{2}-m_{2}\right)}{2 m_{2}}\left[g_{\alpha \beta} \frac{\left(p_{2}+q_{2}\right)_{v}}{2 m_{2}} F_{1}\left(k^{2}\right)+g_{\alpha \beta} \sigma_{v \lambda} \frac{k^{\lambda}}{2 m_{2}} F_{2}\left(k^{2}\right)+\frac{k_{\alpha} k_{\beta}}{4 m_{2}^{2}} \frac{\left(p_{2}+q_{2}\right)_{v}}{2 m_{2}} F_{3}\left(k^{2}\right)+\right. \\
& \left.\left.\frac{k_{\alpha} k_{\beta}}{4 m_{2}^{2}} \sigma_{\nu \lambda} \frac{k^{\lambda}}{2 m_{2}} F_{4}\left(k^{2}\right)\right] \frac{\left(\hat{q}_{2}-m_{2}\right)}{2 m_{2}}\right\} D_{\mu \nu}(k) \hat{\Pi}_{\beta_{1} \sigma \alpha_{1} \rho} L_{\alpha \alpha_{1}} L_{\beta \beta_{1}}\left(g_{\alpha_{1} \beta_{1}}-v_{\alpha_{1}} v_{\beta_{1}}\right), \\
& {\overline{T_{1 \gamma}(\mathbf{p}, \mathbf{q}}}_{F=1}^{j=1 / 2}(\tilde{S}=1)=\frac{Z \alpha}{3} n_{q}^{\delta} n_{p}^{\omega} \operatorname{Tr}\left\{\gamma_{\rho} \frac{1+\hat{v}}{4}\left(\gamma_{\delta}-v_{\delta}\right) \gamma_{5} \frac{\left(\hat{q}_{1}+m_{1}\right)}{2 m_{1}} \Gamma_{\mu} \frac{\left(\hat{p}_{1}+m_{1}\right)}{2 m_{1}} \gamma_{5}\left(\gamma_{\omega}-v_{\omega}\right) \times\right. \\
& \frac{1+\hat{v}}{4} \gamma_{\tau} \frac{\left(\hat{p}_{2}-m_{2}\right)}{2 m_{2}}\left[g_{\alpha \beta} \frac{\left(p_{2}+q_{2}\right)_{v}}{2 m_{2}} F_{1}\left(k^{2}\right)+g_{\alpha \beta} \sigma_{v \lambda} \frac{k^{\lambda}}{2 m_{2}} F_{2}\left(k^{2}\right)+\frac{k_{\alpha} k_{\beta}}{4 m_{2}^{2}} \frac{\left(p_{2}+q_{2}\right)_{v}}{2 m_{2}} F_{3}\left(k^{2}\right)+\right. \\
& \left.\left.\frac{k_{\alpha} k_{\beta}}{4 m_{2}^{2}} \sigma_{\nu \lambda} \frac{k^{\lambda}}{2 m_{2}} F_{4}\left(k^{2}\right)\right] \frac{\left(\hat{q}_{2}-m_{2}\right)}{2 m_{2}}\right\} D_{\mu v}(k) L_{\alpha \alpha_{3}} L_{\beta \beta_{3}} \epsilon_{\rho \beta_{3} \alpha_{1} \beta_{1}} \epsilon_{\tau \alpha_{3} \rho_{1} \omega_{1}}\left(g_{\omega_{1} \beta_{1}}-v_{\omega_{1}} v_{\beta_{1}}\right) .
\end{aligned}
$$

There is also off-diagonal element of the form $<\Psi_{\tilde{S}=0, F=1, M_{F}}\left|V_{1 \gamma, F=1}^{j=1 / 2}\right| \Psi_{\tilde{S}=1, F=1, M_{F}}>$. Omitting other details of the calculation and using (10), (13), (14) we obtain the hyperfine splitting of $2 P_{1 / 2}$ state as follows:

$\Delta E^{h f s}\left(2^{5} P_{1 / 2}-2^{3} P_{1 / 2}\right)=\frac{2 \alpha(Z \alpha)^{3} \mu^{3} \mu_{N}}{27 m_{1} m_{p}}\left[1+\frac{1}{2} a_{\mu}+\frac{m_{1}}{2 m_{2}}-\frac{3 m_{1}}{4 m_{2} F_{2}(0)}\right]=\left\{\begin{array}{l}{ }_{3}^{7} \mathrm{Li}: 210.8960 \mathrm{meV}, \\ { }_{4} \mathrm{Be}:-183.2929 \mathrm{meV}, \\ { }_{5}^{11} \mathrm{~B}: 818.1086 \mathrm{meV},\end{array}\right.$

where we take into account only leading order terms including recoil correction related with nucleus magnetic form factor. At $k^{2}=0$ we have $F_{2}(0)=m_{2} \mu_{N} / Z m_{p}$.

The calculation of hyperfine splitting for the $2^{(2 F+1)} P_{3 / 2}$ state is a more complicated problem, since it is more complicated to construct projection operators for these states. The most simple form is the projection operator on the state with $F=3$. In this case, when the two moments $3 / 2$ are added, we get the state with the maximum total momentum, which is described 
by the tensor $\varepsilon_{\alpha \beta \gamma}$. Then the interaction amplitude averaged over the projections $M_{F}$ can be represented as follows:

$$
\begin{gathered}
\bar{T}_{1 \gamma}(\mathbf{p}, \mathbf{q})_{F=3}^{j=3 / 2}=\frac{3 Z \alpha}{7} n_{q}^{\delta} n_{p}^{\omega} \operatorname{Tr}\left\{\gamma_{\delta_{1}} \frac{1+\hat{v}}{2 \sqrt{2}} \frac{\left(\hat{q}_{1}+m_{1}\right)}{2 m_{1}} \Gamma_{\mu} \frac{\left(\hat{p}_{1}+m_{1}\right)}{2 m_{1}} \frac{1+\hat{v}}{2 \sqrt{2}} \gamma_{\omega_{1}} \times\right. \\
\left.\frac{\left(\hat{p}_{2}-m_{2}\right)}{2 m_{2}} \Gamma_{\alpha \beta}^{v} \frac{\left(\hat{q}_{2}-m_{2}\right)}{2 m_{2}}\right\} D_{\mu v}(k) L_{\alpha \alpha_{1}} L_{\beta \beta_{1}} \hat{\Pi}_{\beta_{1} \delta \delta_{1}, \alpha_{1} \omega \omega_{1}}, \sum_{M_{F}=-3}^{3} \varepsilon_{\alpha \omega \omega_{1}}^{*} \varepsilon_{\beta \delta \delta_{1}}=\hat{\Pi}_{\alpha \omega \omega_{1}, \beta \delta \delta_{1}} .
\end{gathered}
$$

To calculate the interval of the hyperfine structure $\Delta E^{h f s}\left(2^{7} P_{3 / 2}-2^{5} P_{3 / 2}\right)$, it is also necessary to build the potential for the state with $F=2$, which is obtained by adding two $3 / 2$ moments. Acting as in (11), we first represent the state of the nucleus as the result of adding the two moments $\tilde{s}_{2}=1 / 2$ and $l_{2}=1$ and introduce the momentum $\mathbf{j}_{1}=\mathbf{j}+\tilde{\mathbf{s}}_{2}$, which takes values 2 and 1:

$$
\Psi_{S_{2}=3 / 2, j=3 / 2, F=2}=\frac{1}{\sqrt{2}} \Psi_{j_{1}=2, l_{2}=1, F=2}+\frac{1}{\sqrt{2}} \Psi_{j_{1}=1, l_{2}=1, F=2} .
$$

In order to write a projection operator on the state $j_{1}=1$, we will already represent the moment of the muon $j=3 / 2$ as the result of adding two moments: $\mathbf{j}=\mathbf{s}_{1}+\mathbf{l}_{1}$. Given the coefficients of vector addition of moments, we obtain the following expansion:

$$
\Psi_{S_{2}=3 / 2, j=3 / 2, F=2}=\frac{1}{\sqrt{2}} \Psi_{j_{1}=2, l_{2}=1, F=2}+\frac{1}{\sqrt{3}} \Psi_{\left(S=0, l_{1}=1, j_{1}=1\right), l_{2}=1, F=2}+\frac{1}{\sqrt{6}} \Psi_{\left(S=1, l_{1}=1, j_{1}=1\right), l_{2}=1, F=2} .
$$

Performing the addition of individual moments in $F=2$, we obtain the following result for the projection operator on a state with $F=2$ :

$$
\hat{\Pi}_{\alpha \beta, F=2}^{j=3 / 2}=\frac{(1+\hat{v})}{2 \sqrt{6}} \gamma_{5} \varepsilon_{\alpha \beta}+\frac{(1+\hat{v})}{4 \sqrt{6}}\left[-g_{\gamma \alpha} \epsilon_{\beta \alpha_{1} \alpha_{2} \alpha_{3}}+g_{\gamma \beta} \epsilon_{\alpha \alpha_{1} \alpha_{2} \alpha_{3}}+g_{\gamma \alpha_{1}} \epsilon_{\alpha \beta \alpha_{2} \alpha_{3}}\right] v^{\alpha_{2}} \gamma^{\alpha_{1}} \varepsilon^{\gamma \alpha_{3}} \text {. }
$$

The tensor in the right-hand side of (19) contains both the symmetric and antisymmetric parts in the indices $\alpha, \beta$. The same decomposition and addition of individual moments is also used to obtain projection operators on other states of the hyperfine structure with $F=0$ and $F=1$. They have the form:

$$
\begin{gathered}
\hat{\Pi}_{F=0}^{j=3 / 2}=\left[u_{\omega} \bar{v}_{\alpha}(0)\right]_{F=0}^{j=3 / 2}=\frac{1+\hat{v}}{6} \gamma_{5}\left(g_{\omega \alpha}-v_{\omega} v_{\alpha}\right)-\frac{(1+\hat{v})}{12} \gamma^{\lambda} \epsilon_{\lambda \omega \sigma \alpha} v^{\sigma} \\
\hat{\Pi}_{F=1}^{j=3 / 2}=\left[u_{\omega} \bar{v}_{\alpha}(0)\right]_{F=1}^{j=3 / 2}=\frac{1+\hat{v}}{24 \sqrt{5}}\left[-8\left(g_{\omega \alpha}-v_{\omega} v_{\alpha}\right) \gamma_{\alpha_{2}}+2\left(g_{\omega \alpha_{2}} \gamma_{\alpha}+g_{\alpha \alpha_{2}} \gamma_{\omega}\right)-\right. \\
\left.2\left(g_{\alpha_{2} \omega} v_{\alpha}+g_{\alpha \alpha_{2}} v_{\omega}\right)-10 \epsilon_{\omega \alpha \alpha_{3} \alpha_{2}} v^{\alpha_{3}} \gamma_{5}\right] \varepsilon^{\alpha_{2}}
\end{gathered}
$$

In the practical use of (20) - (21), it is convenient to single out the contributions of the symmetric and antisymmetric parts of the projection operators. The general structure of the amplitudes and interaction potentials of particles in these states has the same form as (7), (10), (13), (14), and the intervals of the hyperfine structure themselves are determined by formulas similar to (15):

$\Delta E^{h f s}\left(2^{7} P_{3 / 2}-2^{5} P_{3 / 2}\right)=\frac{\alpha(Z \alpha)^{3} \mu^{3} \mu_{N}}{45 m_{1} m_{p}}\left[1-\frac{1}{4} a_{\mu}+\frac{5 m_{1}}{4 m_{2}}-\frac{15 m_{1}}{8 m_{2} F_{2}(0)}\right]=\left\{\begin{array}{l}{ }_{3}^{7} \mathrm{Li}: 63.8246 \mathrm{meV}, \\ { }_{4} B e:-55.7466 \mathrm{meV}, \\ { }_{5}^{11} \mathrm{~B}: 246.6252 \mathrm{meV},\end{array}\right.$ 


$$
\begin{gathered}
\Delta E^{h f s}\left(2^{5} P_{3 / 2}-2^{3} P_{3 / 2}\right)=\frac{2 \alpha(Z \alpha)^{3} \mu^{3} \mu_{N}}{135 m_{1} m_{p}}\left[1-\frac{1}{4} a_{\mu}+\frac{5 m_{1}}{4 m_{2}}-\frac{15 m_{1}}{8 m_{2} F_{2}(0)}\right]=\left\{\begin{array}{l}
{ }_{3}^{7} L i: 42.5497 \mathrm{meV}, \\
9 B e:-37.1644 \mathrm{meV}, \\
{ }_{4}^{11} B: 164.4168 \mathrm{meV},
\end{array}\right. \\
\Delta E^{h f s}\left(2^{3} P_{3 / 2}-2^{1} P_{3 / 2}\right)=\frac{\alpha(Z \alpha)^{3} \mu^{3} \mu_{N}}{135 m_{1} m_{p}}\left[1-\frac{1}{4} a_{\mu}+\frac{5 m_{1}}{4 m_{2}}-\frac{15 m_{1}}{8 m_{2} F_{2}(0)}\right]=\left\{\begin{array}{l}
{ }_{3}^{7} L i: 21.2932 \mathrm{meV}, \\
9 \\
{ }_{4} B e:-18.5822 \mathrm{meV}, \\
{ }_{5}^{11} B: 82.2084 \mathrm{meV},
\end{array}\right.
\end{gathered}
$$

The numerical values of the contributions (15), (22)-(24) are large. Therefore, to increase the accuracy of calculations, it makes sense to consider a number of corrections to these formulas.

However, it is useful to consider another approach to solving this problem in the coordinate representation, which is widespread [8]. Since in muon ions we encounter nuclei of different spins, it is necessary to have a two-particle Hamiltonian for electromagnetically interacting particles of arbitrary spin. Some time ago, the task of constructing such an effective Hamiltonian was solved in $[17,18]$ in connection with the calculation of gyromagnetic factors of bound particles. To calculate the hyperfine structure of the spectrum of P-levels, it is necessary to use the following term from this Hamiltonian:

$$
\Delta H^{h f s}=\frac{Z \alpha g_{N}}{2 m_{1} m_{2} r^{3}}\left[1+\frac{m_{1}}{m_{2}}-\frac{m_{1}}{m_{2} g_{N}}\right]\left(\mathbf{L s}_{2}\right)-\frac{Z \alpha\left(1+a_{\mu}\right) g_{N}}{2 m_{1} m_{2} r^{3}}\left[\mathbf{s}_{1} \mathbf{s}_{2}-3\left(\mathbf{s}_{1} \mathbf{r}\right)\left(\mathbf{s}_{2} \mathbf{r}\right)\right],
$$

where the gyromagnetic factor of the nucleus $g_{N}=F_{1}(0) / s_{2}=2 m_{2} \mu_{N} / 3 Z m_{p}$ and nucleus magnetic moment is taken in nuclear magnetons. To calculate the relative level arrangement, a fine part of the Hamiltonian is also necessary which leads to

$$
E^{f s}=\frac{(Z \alpha)^{4} \mu^{3}}{16 m_{1} m_{2}}\left[1+\frac{m_{2}}{2 m_{1}}+a_{\mu}\left(1+\frac{m_{2}}{m_{1}}\right)\right]=\left\{\begin{array}{l}
{ }_{3}^{7} \mathrm{Li}: 747.8581 \mathrm{meV}, \\
{ }_{4} B e: 2372.2215 \mathrm{meV}, \\
{ }_{5}^{11} B: 5804.9674 \mathrm{meV}
\end{array}\right.
$$

The hyperfine part of the Hamiltonian includes two operators $T_{1}=\mathbf{L s}_{2}, T_{2}=\mathbf{s}_{1} \mathbf{S}_{2}-$ $3\left(\mathbf{s}_{1} \mathbf{n}\right)\left(\mathbf{s}_{2} \mathbf{n}\right)$. Diagonal in j matrix elements contribute to the hyperfine structure in the form:

$$
E\left(2^{2 F+1} P_{j}\right)=\frac{\alpha(Z \alpha)^{3} \mu^{3} \mu_{N}}{72 m_{1} m_{p}}\left[\bar{T}_{1}+\frac{m_{1}}{m_{2}} \bar{T}_{1}-\frac{3 m_{1}}{2 m_{2} F_{2}(0)} \bar{T}_{1}-\left(1+a_{\mu}\right) \bar{T}_{2}\right] .
$$

The calculation of matrix elements $\bar{T}_{1}$ and $\bar{T}_{2}$ is carried out using the basic formulas from [8] (see Appendix A). As a result, the position of energy levels $2^{2 F+1} P_{j}$ is determined by the following expressions:

$$
\begin{aligned}
& E\left(2^{7} P_{3 / 2}\right)=\tilde{E}^{f s}+\frac{\alpha(Z \alpha)^{3} \mu^{3} \mu_{N}}{60 m_{1} m_{p}}\left[1-\frac{a_{\mu}}{4}+\frac{5 m_{1}}{4 m_{2}}-\frac{15 m_{1}}{8 m_{2} F_{2}(0)}\right]=\left\{\begin{array}{l}
7 \mathrm{Li}: 798.0748 \mathrm{meV}, \\
{ }_{9} \mathrm{Be}: 2340.5274 \mathrm{meV}, \\
{ }_{1}^{11} B: 6020.4780 \mathrm{meV},
\end{array}\right. \\
& E\left(2^{5} P_{3 / 2}\right)=\tilde{E}^{f s}-\frac{\alpha(\mathrm{Z} \alpha)^{3} \mu^{3} \mu_{N}}{180 m_{1} m_{p}}\left[1-\frac{a_{\mu}}{4}+\frac{5 m_{1}}{4 m_{2}}-\frac{15 m_{1}}{8 m_{2} F_{2}(0)}\right]=\left\{\begin{array}{l}
{ }_{3}^{7} \mathrm{Li}: 734.2502 \mathrm{meV}, \\
{ }_{4} B e: 2396.2740 \mathrm{meV}, \\
{ }_{5} B: 5773.8528 \mathrm{meV},
\end{array}\right.
\end{aligned}
$$




$$
\begin{gathered}
E\left(2^{3} P_{3 / 2}\right)=\tilde{E}^{f s}-\frac{11 \alpha(Z \alpha)^{3} \mu^{3} \mu_{N}}{540 m_{1} m_{p}}\left[1-\frac{a_{\mu}}{4}+\frac{5 m_{1}}{4 m_{2}}-\frac{15 m_{1}}{8 m_{2} F_{2}(0)}\right]=\left\{\begin{array}{l}
{ }_{3}^{7} \mathrm{Li}: 691.7005 \mathrm{meV}, \\
9 \\
4 \\
{ }_{1} B e: 2433.4383 \mathrm{meV}, 5609.4360 \mathrm{meV},
\end{array}\right. \\
E\left(2^{1} P_{3 / 2}\right)=\tilde{E}^{f s}-\frac{\alpha(Z \alpha)^{3} \mu^{3} \mu_{N}}{36 m_{1} m_{p}}\left[1-\frac{a_{\mu}}{4}+\frac{5 m_{1}}{4 m_{2}}-\frac{15 m_{1}}{8 m_{2} F_{2}(0)}\right]=\left\{\begin{array}{l}
{ }_{3}^{7} \mathrm{Li}: 670.4256 \mathrm{meV}, \\
9 \\
{ }_{4}^{11} \mathrm{Be}: 2452.0205 \mathrm{meV},
\end{array}\right. \\
E\left(2_{5}^{5} P_{1 / 2}\right)=\frac{\alpha(Z \alpha)^{3} \mu^{3} \mu_{N}}{36 m_{1} m_{p}}\left[1+\frac{a_{\mu}}{2}+\frac{m_{1}}{2 m_{2}}-\frac{3 m_{1}}{4 m_{2} F_{2}(0)}\right]=\left\{\begin{array}{l}
{ }_{3}^{7} \mathrm{Li}: 79.0860 \mathrm{meV}, \\
{ }_{4}^{9} B e:-68.7348 \mathrm{meV}, \\
{ }_{5}^{11} \mathrm{~B}: 306.7907 \mathrm{meV},
\end{array}\right. \\
E\left(2^{3} P_{1 / 2}\right)=-\frac{5 \alpha(Z \alpha)^{3} \mu^{3} \mu_{N}}{108 m_{1} m_{p}}\left[1+\frac{a_{\mu}}{2}+\frac{m_{1}}{2 m_{2}}-\frac{3 m_{1}}{4 m_{2} F_{2}(0)}\right]=\left\{\begin{array}{l}
{ }_{3}^{7} \mathrm{Li}:-131.8100 \mathrm{meV}, \\
{ }_{4} \mathrm{Be}: 114.5581 \mathrm{meV}, \\
{ }_{5}^{11} \mathrm{~B}:-511.3179 \mathrm{meV},
\end{array}\right.
\end{gathered}
$$

where we add (26) to fix the relative position of sublevels. The obtained expressions (30)(33) which contain the factor $1 / g_{N}$ coming from the hyperfine interaction Hamiltonian give the hyperfine splitting coinciding with (15), (22)-(24).

Table 1. Matrix elements of hyperfine structure of $2 P$-states in muonic ions $\mathrm{Li}, \mathrm{Be}, \mathrm{B}$ in first order perturbation theory. The first, second and third lines correspond to lithium, beryllium and boron.

\begin{tabular}{|c|c|c|c|c|c|c|}
\hline Contribution & $\begin{array}{c}2^{3} P_{1 / 2}, \\
\mathrm{meV}\end{array}$ & $\begin{array}{c}2^{5} P_{1 / 2}, \\
\mathrm{meV}\end{array}$ & $\begin{array}{c}2^{1} P_{3 / 2}, \\
\mathrm{meV}\end{array}$ & $\begin{array}{c}2^{3} P_{3 / 2}, \\
\mathrm{meV}\end{array}$ & $\begin{array}{c}2^{5} P_{3 / 2}, \\
\mathrm{meV}\end{array}$ & $\begin{array}{c}2^{7} P_{3 / 2}, \\
\mathrm{meV}\end{array}$ \\
\hline $\begin{array}{c}\text { Leading order } \\
\alpha^{4} \text { correction }\end{array}$ & -131.8100 & 79.0860 & 670.4256 & 691.7005 & 734.2502 & 798.0748 \\
& 114.5581 & -68.7348 & 2452.0205 & 2433.4383 & 2396.27400 & 2340.5274 \\
& -511.3179 & 306.7907 & 5527.2276 & 5609.4360 & 5773.8528 & 6020.4780 \\
\hline Quadrupole & 0 & 0 & -186.9598 & -37.3920 & 112.1759 & -37.3920 \\
correction & 0 & 0 & 583.5774 & 116.7155 & -350.1465 & 116.7155 \\
of order $\alpha^{4}$ & 0 & 0 & 882.8935 & 176.5787 & -529.7361 & 176.5787 \\
\hline VP correction & -0.5701 & 0.3420 & -0.2784 & -0.2042 & -0.0557 & 0.1671 \\
of order $\alpha^{5}$ & 0.6710 & -0.4026 & 0.3441 & 0.2523 & 0.0689 & -0.2065 \\
& -3.6909 & 2.2146 & -1.9209 & -1.4087 & -0.3842 & 1.1526 \\
\hline Quadrupole, & 0 & 0 & -0.6573 & -0.1315 & 0.3944 & -0.1315 \\
VP correction & 0 & 0 & 2.7276 & 0.5455 & -1.6365 & 0.5455 \\
of order $\alpha^{5}$ & 0 & 0 & 5.0232 & 1.0046 & -3.0140 & 1.0046 \\
\hline Relativistic & -0.1289 & 0.0773 & -0.0115 & -0.0084 & -0.0023 & 0.0069 \\
correction & 0.1964 & -0.1178 & 0.0176 & 0.0129 & 0.0035 & -0.0105 \\
of order $\alpha^{6}$ & -1.3686 & 0.8212 & -0.1223 & -0.0897 & -0.0245 & 0.0734 \\
\hline VP correction & -0.0011 & -0.0007 & -0.0004 & -0.0003 & -0.0001 & 0.0002 \\
of order $\alpha^{6}$ & 0.0011 & -0.0007 & 0.0005 & 0.0003 & 0.0001 & -0.0003 \\
& -0.0054 & 0.0032 & -0.0023 & -0.0017 & -0.0005 & 0.0014 \\
\hline Structure & -0.0784 & 0.0471 & -0.0008 & -0.0007 & -0.0004 & -0.0001 \\
correction & 0.1295 & -0.0777 & 0.0018 & 0.0015 & 0.0008 & -0.0001 \\
of order $\alpha^{6}$ & -0.8292 & 0.4975 & -0.0050 & -0.0043 & -0.0028 & -0.0007 \\
\hline Summary & -132.5885 & 79.5517 & 482.5174 & 653.9634 & 845.9732 & 760.7254 \\
contribution & 114.2141 & -69.3336 & 3038.6896 & 2550.9663 & 2044.5643 & 2457.5710 \\
& -517.2120 & $310.3272 \mathrm{~b}$ & 6413.0938 & 5785.8732 & 5240.6907 & 6199.2880 \\
\hline
\end{tabular}




\section{Conclusion}

Knowledge of the hyperfine splitting of the P levels is necessary for calculating the transition frequencies between energy levels $2 \mathrm{~S}$ and $2 \mathrm{P}$. In this work we investigate the hyperfine structure of energy levels in muonic ions of lithium, beryllium and boron on the basis quasipotential method in quantum electrodynamics. Our calculation contains the leading or$\operatorname{der} \alpha^{4}$ contribution and corrections of orders $\alpha^{5}$ and $\alpha^{6}$ to the vacuum polarization, nucleus structure, quadrupole interaction and relativism. These corrections have significant numerical value because of the factor proportional to the nucleus charge. Numerical results are written in Tables 1.

This work continues our investigation of P-wave part of the energy spectrum in light muonic atoms which was begun in $[8,19-21]$. Taking nucleus of spin $3 / 2$ we obtain new results in the study of hyperfine structure which are as follows:

1. The method of projection operators for spin nuclei $3 / 2$ in the momentum representation is developed for construction of muon-nucleus interaction operator for various states.

2. The corrections of order $O\left(\alpha^{5}\right)$ on vacuum polarization are calculated in the hyperfine structure of the P-states, including the quadrupole interaction.

3. The corrections of order $O\left(\alpha^{6}\right)$ on vacuum polarization and nucleus structure are obtained. Relativistic corrections are also taken into account in the framework of the relativistic Dirac equation.

The work is supported by Russian Science Foundation (grant No. RSF 18-12-00128) and Russian Foundation for Basic Research (grant No. 18-32-00023) (F.A.M.).

\section{References}

[1] R. Pohl, Jour. Phys. Soc. Japan 85, 091003 (2016)

[2] S. Schmidt et al. J. Phys. Conf. Ser. 1138, 012010 (2018)

[3] G.W.F. Drake and L.L. Byer, Phys. Rev. A 32, 713 (1985)

[4] R. Swainson and G.W.F. Drake, Phys. Rev. A 34, 620 (1986)

[5] A.A. Krutov et al., Phys. Rev. A 94, 062505 (2016)

[6] A.E. Dorokhov, A.A. Krutov, A.P. Martynenko et al., Phys. Rev. A 98, 042501 (2018)

[7] A.E. Dorokhov et al., Phys. Part. Nucl. 50, 635 (2019)

[8] R.N. Faustov et al., Phys. Rev. A 92, 052512 (2015)

[9] R.N. Faustov et al., Phys. Rev. A 90, 012520 (2014)

[10] J.G. Körner, M. Krämer and D. Pirjol, Prog. Part. Nucl. Phys. 33, 787 (1994)

[11] F.A. Berends et al., Nucl. Phys. B 154, 261 (1979)

[12] J.H. Kühn, J. Kaplan and El G.Q. Safiani, Nucl. Phys. B 157, 125 (1979)

[13] J.A.M. Vermaseren, e-preprint arXiv:math-ph/0010025

[14] S. Nozawa and D.B. Leinweber, Phys. Rev. D 42, 3567 (1990)

[15] T.M. Aliev, K. Azizi, and M. Savci, Phys. Lett. B 681, 240 (2009)

[16] S. Deser, A. Waldron and V. Pascalutsa, Phys. Rev. D 62, 105031 (2000)

[17] K. Pachucki, Phys. Rev. A 78, 012504 (2008)

[18] M.I. Eides and T.J.S. Martin, Phys. Rev. Lett. 105, 100402 (2010)

[19] E.N. Elekina and A.P. Martynenko, Phys. Atom. Nucl. 73, 1828 (2010)

[20] A.P. Martynenko, Phys. Atom. Nucl. 71, 125 (2008)

[21] A.E. Dorokhov, N.I. Kochelev, A.P. Martynenko et al. Eur. Phys. Jour. A 54, 131 (2018) 